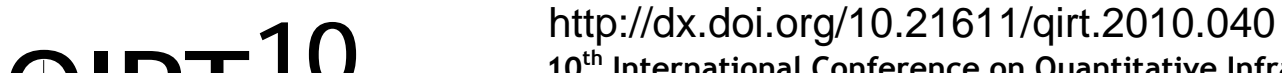 \\ $10^{\text {th }}$ International Conference on Quantitative InfraRed Thermography \\ July 27-30, 2010, Québec (Canada)
}

\section{Analysis of Fourier images of thermograms for purposes of machine diagnostics}

\author{
by M. Fidali* \\ *KPKM Dept., Silesian University of Technology, Poland, Gliwice, Konarskiego 18a str., marek.fidali@polsl.pl
}

\begin{abstract}
In the paper results of analysis of Fourier images were presented. Fourier images called also F-images were results of Fourier analysis of thermovision images of a laboratory model of rotating machinery. The thermovision images presenting different technical states of the investigated object were acquired during an active diagnostic experiment. In order to estimate F-images three estimators were proposed. These estimators allowed to evaluate features represented by the mean value of a specific area of the F-image. The estimation results depend on estimator parameters which values should be determined in advance. Obtained result shows that a proposed way of estimation of thermovision images could be useful for identification of machine technical state.
\end{abstract}

\section{Introduction}

Thermographic measurements find broad application in maintenance and technical state assessment of machinery, apparatus and industrial processes [10]. Technological progress and decreasing prices of industrial thermovision cameras make their application to continuous monitoring and assessing a technical state of machines and devices even more possible. Thermal monitoring and diagnosing of machines requires extraction of relevant diagnostic information coded in thermographic images. The extraction of important diagnostic data can be performed with the use of properly fitted methods of thermovision image processing, analysis and recognition. Research connected with the application of image processing and analysis methods to thermovision diagnostics has been carried out in Department of Fundamentals of Machinery Design for a few years $[3,4,5]$. During the research a formalised method of thermovision data analysis has been proposed.

According to the method a sequence of thermograms acquired during a continuous observation of a machine is treated as a multidimensional thermovision signal which is transformed to a set of one-dimensional relevant diagnostic signals. Determination of this relevant signal on the basis of the thermographic signal is connected with a search for such relevant features of thermographic signal which values describe as many machine technical states as possible in the most efficient way. In reality a few relevant features are necessary to achieve an optimal performance of machine technical state classification. In order to analyse a multidimensional thermovision signal and extract useful diagnostic data different representations of IR images can be taken into consideration. Examples are thermovision images that are results of Fourier or Wavelets transformations. The article deals with analysis of F-images obtained as result of 2D Fourier analysis of thermovision images.

\section{2D Fourier transform of infrared images}

Fourier transform and the efficient computing algorithm (FFT) are well known and widely described in many publications [1],[2],[7],[9],[8]. Two-dimensional version of the transform is a very useful tool, both for the spectral analysis of the image content and as an aid in the construction of imaging filters. The result of Fourier analysis of an image is two-dimensional spectrum which could be represented by two images of magnitude and phase called also F-images or magnitudegrams and phasegrams. The arrangement of 2D Fourier transform images localizes the low frequency components at the image corners Fig. 1a. Such representation of the frequency components is inconvenient in its interpretation thus in order to visualise the Fimage spatial components in easier way, the content of F-images is rearranged Fig. 1b. Zero frequency component (the d.c. component represents the average value of the samples) is in the F-image centre, with frequency increasing towards the edge of the image [8],[9]. Such F-image visualisation does not change the frequency domain information but only the way it is displayed.

An important property of the Fourier transform is the independence of the position of features within the image. The decomposition into spatial frequency does not depend on the position of features within the image. If we shift all the features by a fixed amount, or acquire the image from a different position, the magnitude of its Fourier transform does not change [8]. This property is known as the shift invariance. 
a)

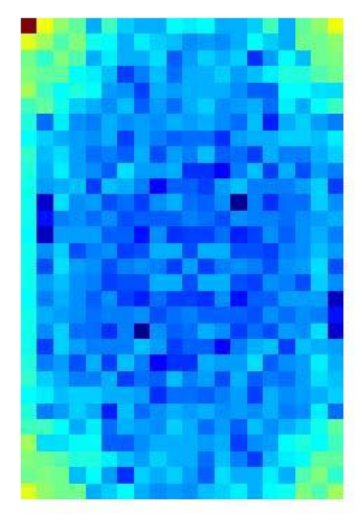

b)

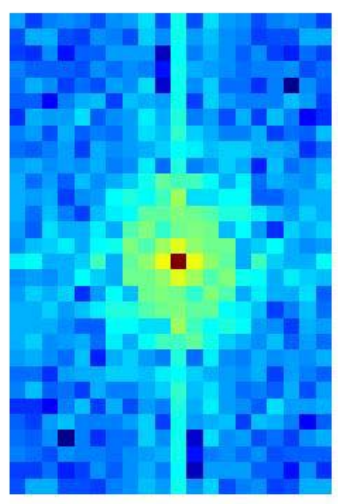

Fig. 1. An exemplary F-images of amplitude before (a) and after (b) shifting frequency components

2D Fourier transform in comparison to the 1D version produces data (images) which are difficult for interpretation. Determination of relations between visual information in the image and frequency components of Fourier images is rather complicated especially in the case of thermovision images. Difficulties in interpretation of F-images keep the 2D Fourier analysis still an interesting tool of images analysis and open wide possibilities in the search for methods of F-image processing, analysis and recognition.

\section{F-images estimators}

The method of F-images analysis in most cases depends on the nature and goal of the research.

Theoretically, frequency components on the shifted F-images are distributed symmetrically and in many cases of the analysis it is enough to consider one quarter of the magnitudograms and/or two adjacent quarters of the phasegrams [9]. Practically, for the analysis is considered an entire F-image what is shown in different papers [6],[9],[11]. This approach is most convenient for F-image interpretation purposes because the symmetry in the F-image components generates specific patterns which shapes and locations depend on a content of the original image being transformed.

One can observe that in F-images evaluated on the basis of thermograms recorded in different moments of machine operation, there are patterns which change along time and depend on the machine state. On the basis of a preliminary visual investigation of evaluated F-images one concluded that in some cases the frequency components of F-images generated shapes similar to stars which centra overlapped the centre of the F-image and amplitudes decreased when the frequency was growing (Fig. 2). Horizontal and/or vertical distributions of F-pixel values along the image axes of symmetry were also observed. Such patterns and the observed regularity allowed to define estimators of F-images which based on mean values of frequency components in specific areas.

a)

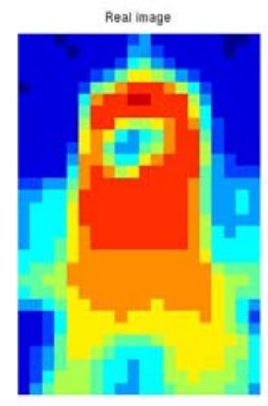

b)

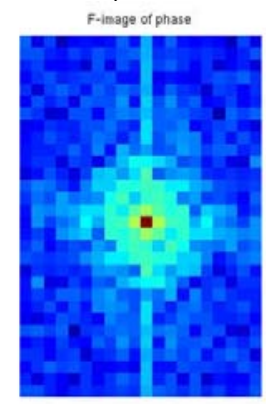

c)

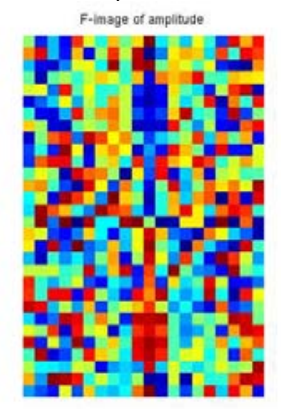

Fig. 2. An exemplary thermovision image (a) and its F-images of magnitude (b) and phase (c) obtained on the basis of 2D Fourier analysis 
According to the visual investigation and considering the way F-image analysis were presented in [6], the following quantitative estimators of the F-images were proposed.

- $\quad$ VFP - Vertical Fourier Power (Fig. 3a)

$$
V F P=\sum_{x=\frac{X-W}{2}+1}^{\frac{X+W}{2}} \sum_{y=1}^{Y} F(x, y)
$$

- $\quad$ HFP - Horizontal Fourier Power (Fig. 3b)

$$
H F P=\sum_{x=1}^{X} \sum_{x=\frac{Y-H}{2}+1}^{\frac{Y+H}{2}} F(x, y)
$$

- $\quad$ CFP - Circular Fourier Power (Fig. 3c)

$$
C F P=\sum_{r=\frac{X-D}{2}+1^{2}}^{\frac{X+D}{2}} \sum_{1 \leq x^{2}+y^{2} \leq r} F(x, y)
$$

where:

$X$ - F-image width in pixels

$Y$ - F-image height in pixels

$\mathrm{W}$ - width of the considered central vertical strip of the F-image in pixels

$\mathrm{H}$ - width of the considered central horizontal strip of the F-image in pixels

$D$ - diameter of the considered centralized circle of the F-image in pixels

$\mathrm{X}=1,2, \ldots, \mathrm{X}$ - pixel indicating image width

$y=1,2, \ldots, Y$ - pixel indicating image height

As indicated in Fig. 3, the proposed estimators are the mean values of frequency components calculated for rectangular and circular areas of F-images placed in their centre. One can easily observe that the estimator values strongly depend on parameters \$W\$, \$H and \$D which should be established in advance.

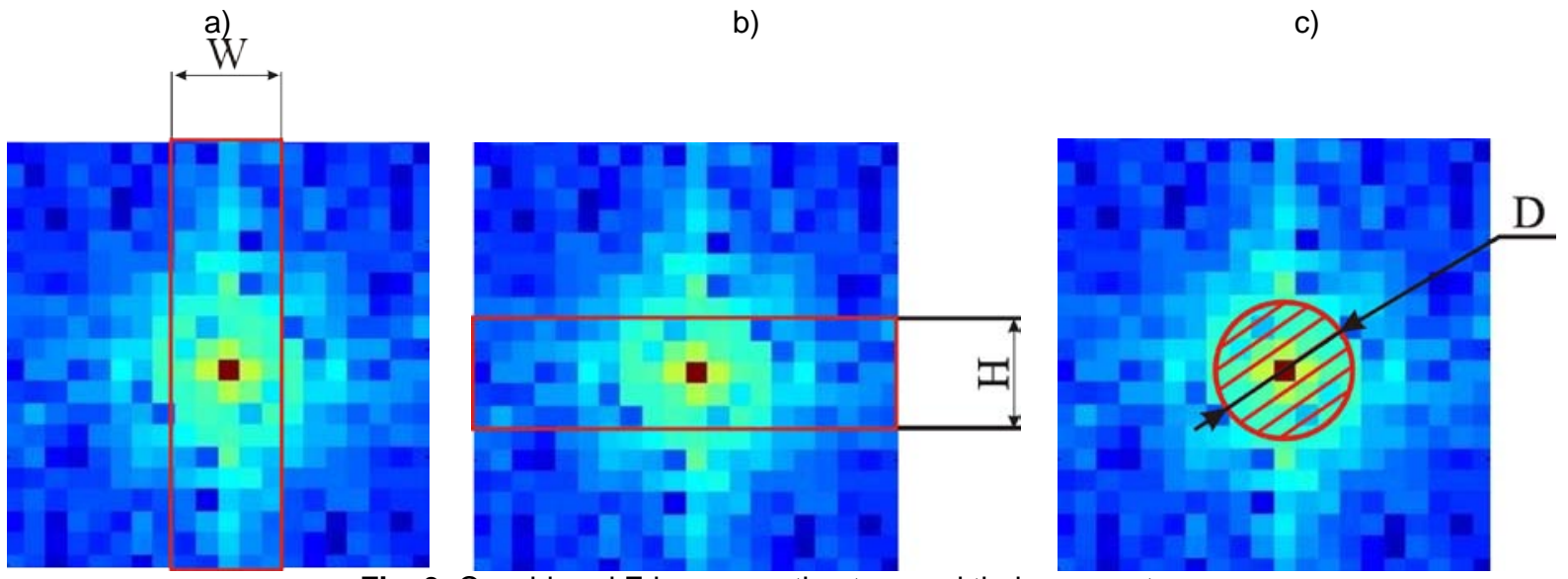

Fig. 3. Considered F-images estimators and their parameters

\section{Results of F-images analysis}

In order to perform the estimation of F-images of a thermogram it was necessary to gather suitable data. An active diagnostic experiment was carried out with the use of a laboratory stand located in the Laboratory of Technical Diagnostics of the Department of Fundamentals of Machinery Design. The stand consisted of a laboratory model of rotating machinery and a thermovision system (Fig. 4). During the experiment a sequence (thermovision signal) of 840 thermovision images presenting the object operating in different technical states was recorded. 


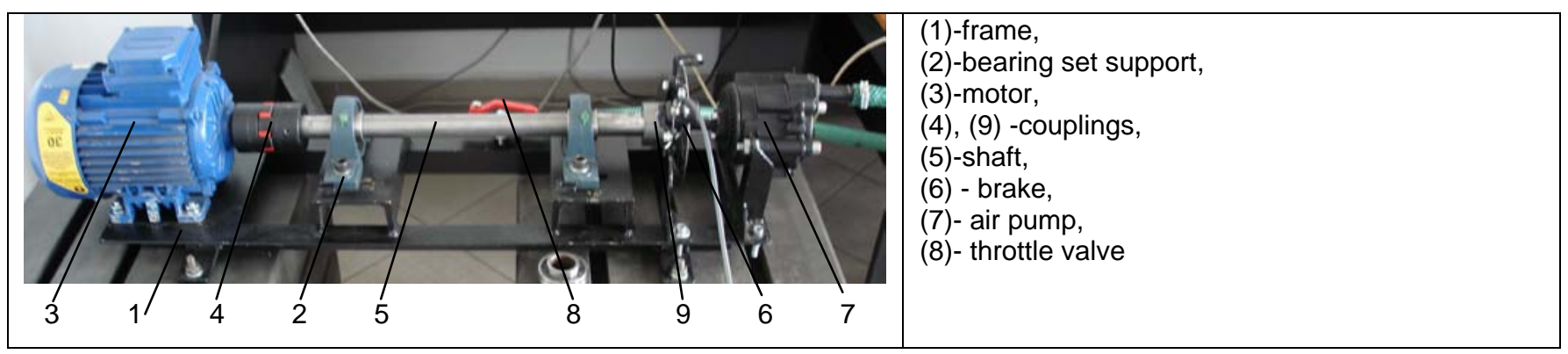

The following technical states were simulated:

Fig. 4. Investigated object

S1 - machine without faults--240 images

S2 - 50\% throttling of the air pump--120 images

S3 - 90\% throttling of the air pump--120 images

S4 - 901\% throttling of the air pump and clearance of the second bearing mounting--120 images

S5 - load of the disk brake--120 images

S6 - faulty bearing no 2--120 images.

It is necessary to point out that technical states S2 and S3 were very similar and were simulated purposely in order to check whether it was possible to notice a weak change of the technical state on the basis of the considered diagnostic signals. Such a small change in the technical state were also desirable for testing ability of the classifiers recognizing machine technical states difficult to be distinguished.

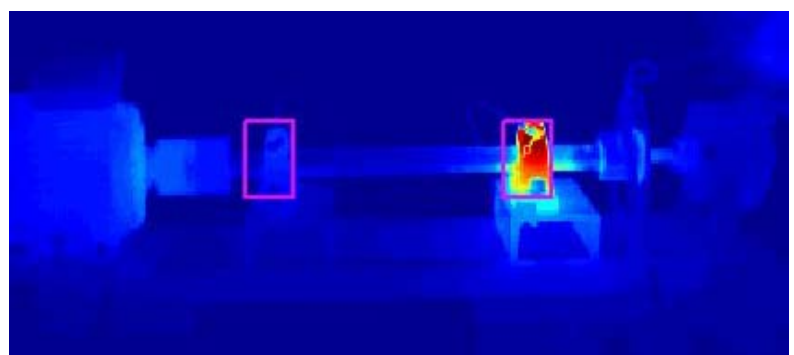

Fig. 5. Thermovision image of the operating laboratory stand, with marked ROIs of the first (left, ROI1) and the second (right, ROI2) bearings

The thermovision images acquired during the experiment were processed. The first operation was selection of interesting areas in the thermograms. One decided to select two Regions Of Interest (ROI1 and ROI2) representing the bearing housings (Fig. 5). Those ROls were interesting due to two reasons. Firstly, the most of the machine failures are visible in bearings operation and secondly the construction and shape of both the bearings housing were the same. One expected that changes in the machine technical state affect changes of bearings temperature and should be revealed in the sequence of thermographic images. Taking into account two ROls presenting a view of the same type of the bearing housing could be beneficial for the verification of different image features.

The recorded sequence of thermovision images represented by the selected ROIs was transformed into frequency domain with the use of 2D Fourier transform. One obtained two Fourier images (F-images) of amplitude (magnitudegrams) and phase (phasegrams) for every considered image. F-images, both amplitude and phase were analysed with the use of developed estimators.

In Fig. 6. - Fig. 8 an exemplary plot of values of Vertical (VFP), Horizontal (HFP) and Circular Fourier Power (CFP) as a function of consecutive recorded thermogram indexes are presented. Such functions are examples of diagnostic signals. Estimator values were calculated for F-images of amplitude for both considered ROls. During calculation of F-images estimators one considered different values of estimator parameters $\mathrm{W}, \mathrm{H}, \mathrm{D}$ in order to check how those parameters influence on diagnostic signals.

In the plots limits of simulated technical states have been indicated in order to identify places where changes of technical states should occur. It can be observed that identification of changes of the machine technical state on the basis of calculated diagnostic signal is possible. The ability to recognise technical states depends on considered ROI. In case of ROI2 which represented temperature of more loaded bearing housing dynamics of diagnostics signals, during state change, is higher than in case of the first bearing housing represented in ROl1. States no 5 and 6 are easily recognizable in plots 
generated from both ROIs. The change between states S1 to S2 in case of ROI2 is also clearly visible. Recognition of changes in diagnostic signals when machine passed from state S1 to S2 for ROI1 and from S2 to S3 and from S3 to S4 for both ROIs is rather difficult and ambiguous. Plots show that ability to identification of these weak recognizable states, depends on applied estimator parameters. In case of VFP as well as HFP lower values of estimator parameters slightly improve visual state recognitions. Visual discrimination of the machine states in case of CFP plots generated for different parameter of the estimator indicates better results are in case of high values.
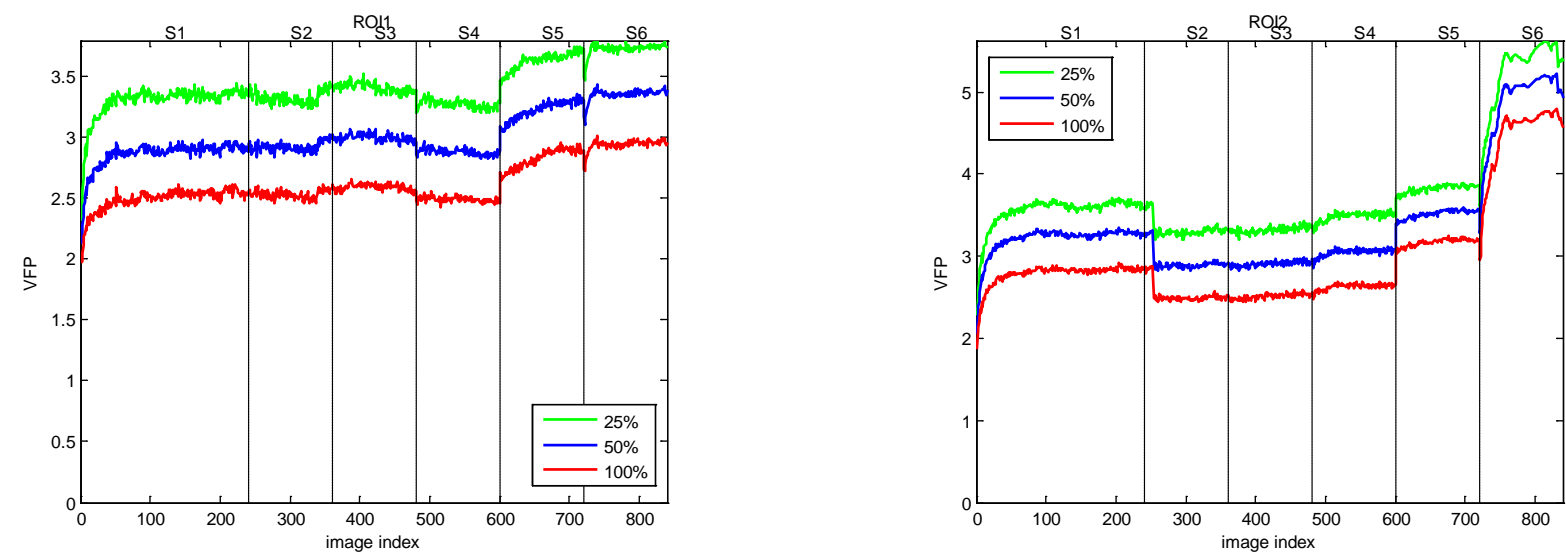

Fig. 6. Plot of VFP values estimated for ROI1 and ROI2 as a function of recorded Ir image indexes
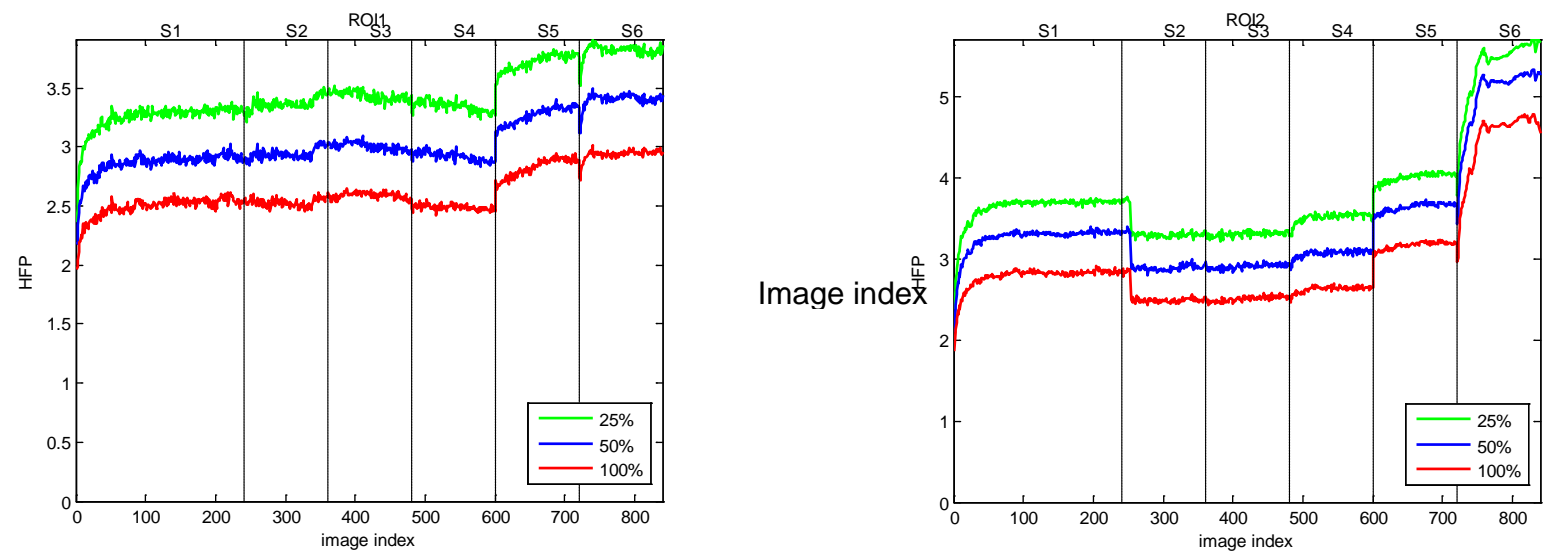

Fig. 7. Plot of HFP values estimated for ROI1 and ROI2 as a function of recorded Ir image indexes
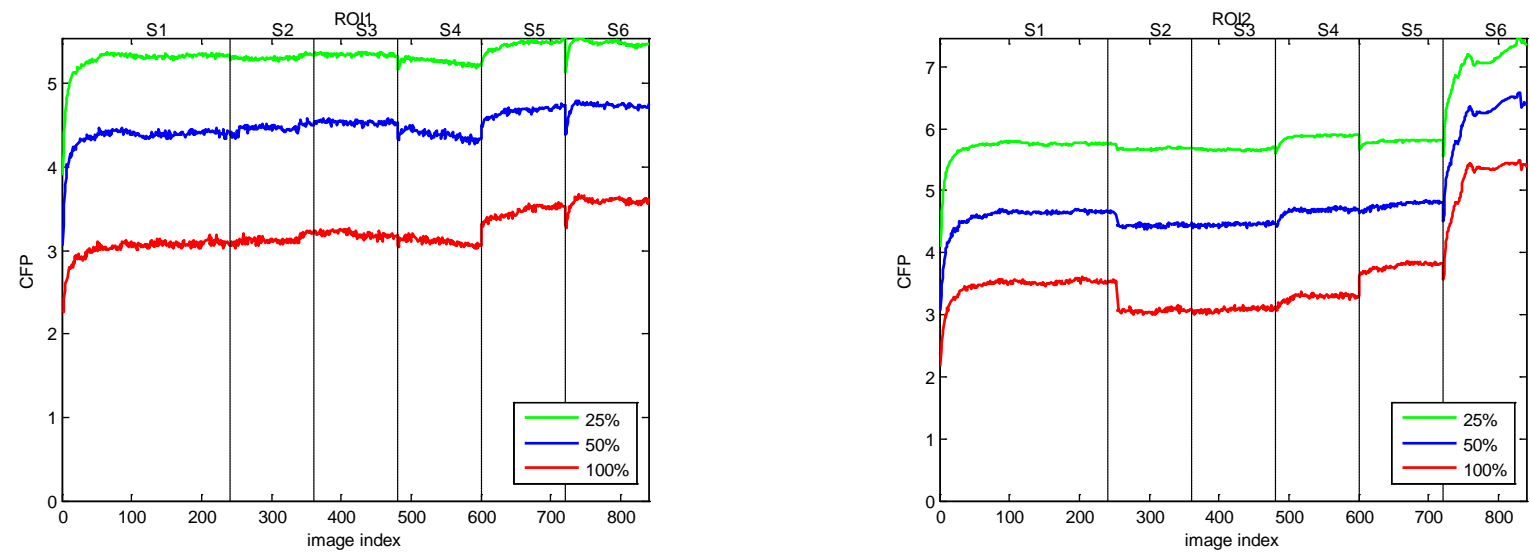

Fig. 8. Plot of CFP values estimated for ROI1 and ROI2 as a function of recorded Ir image indexes 


\section{Conclusions}

The article deals with the application of F-images to assessment of machine technical state. In order to estimate Fimages estimators were proposed. Values of the estimators depend on spatial distribution of F-image frequency components. Calculation of estimator values for thermograms recorded during continuous thermovision observation of the monitored machine, allowed us to generate diagnostic signals which could be used for further analysis. Results of the analysis of generated diagnostic signals showed that proposed estimators of F-image of thermograms could be useful for identification of changes of machine technical state. Sensitivity of F-image estimators to technical state changes depended on value of estimators parameters. In the case of the estimators HFP and VFP the best technical state discrimination could be performed for small values of the estimator parameter. The change of parameters of CFP estimators from low to high showed that ability to discrimination of machine technical are slightly better for higher values. It is important to point out that assessment of the generated diagnostic signals was performed subjectively on the basis of visual investigation of the plots. Objective assessment of the obtained results will be subject of further research.

\section{REFERENCES}

[1] Acharya T., Ajoy R.: Image Processing Principles and Applications. A John Wiley \& Sons, 2005.

[2] Bracewell R., ed., The Fourier Transform and Its Applications. New York: McGraw-Hill, 1965.

[3] Fidali M.: Application of analysis of thermographic images to machine state assessment. In Recent Advances in Mechatronics. Jabłoński R., Turkowski M., and Szewczyk R., eds., , Springer Berlin Heidelberg, 2007.

[4] Fidali M.: An idea of continuous thermographic monitoring of machinery, in QIRT 2008 Proceedings, 9th International Conference on Qualitative InfraRed Thermography, July 2-5, Kraków, Poland, 2008.

[5] Fidali M., Bzymek A.: Identification of changes of machine technical state on the basis of PCA of sequence of thermographic images. Diagnostyka, no. 3(51), pp. 45_48, 2009.

[6] Fukushima M., Ogawa K., Kubota T., Hisa N.: Quantitative tissue characterization of diffuse liver diseases from ultrasound images by neural network. in Nuclear Science Symposium, vol. 2, pp. 1233-1236, 1998.

[7] Gonzales R., Wintz P.: Digital Image Processing. Addison-Wesley Publishing Company, 1987.

[8] Nixon S. M., Aguado S.: Feature Extraction and Image Processing. Newnes, 2002.

[9] Tadeusiewicz R., Korohoda P.: Komputerowa analiza i przetwarzanie obrazów. Wydawnictwo Fundacji Postępu Telekomunikacji, Kraków 1997. (in Polish)

[10] Thomas R. A.: The thermography monitoring handbook. Machine \& systems condition monitoring series, Oxford, UK: Coxmoor Pub., 1999.

[11] Tunak M., Linka A.: Analysis of planar anisotrophy of fibre system by using 2D Fourier transform. Fibres \& Textiles in Eastern Europe, vol. 15, pp. 86_90, December 2007. 